Im „Journal Club" werden Originalarbeiten aus der internationalen Fachliteratur referiert und kommentiert.

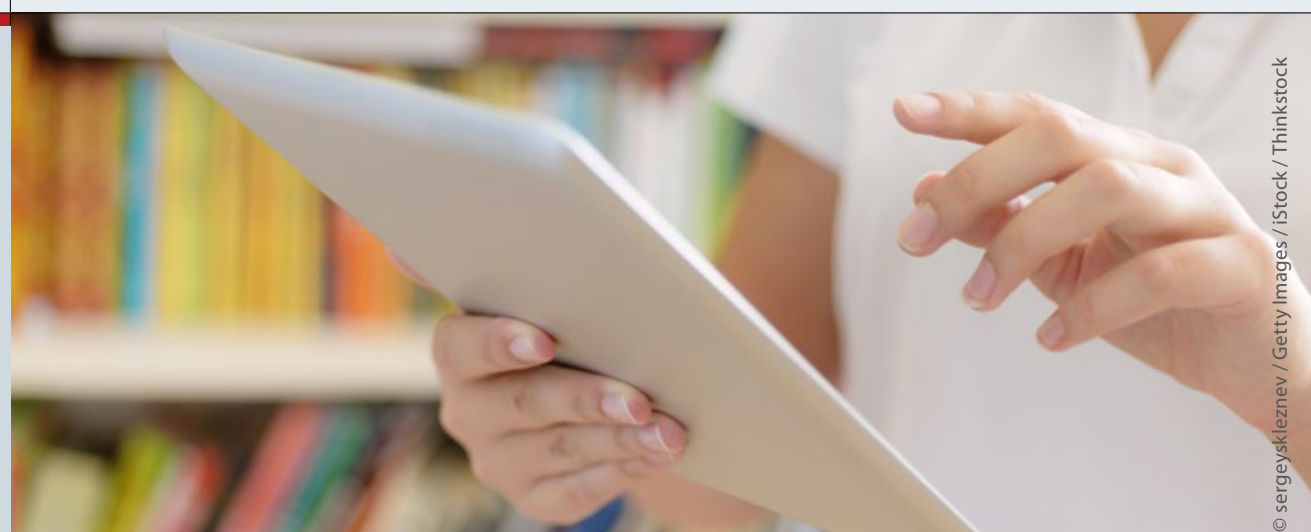

\section{Olanzapin hilft gegen Zytostatika-Erbrechen}

\author{
Für das Neuroleptikum Olanzapin ergibt sich eine neue Indikation: Die \\ Einnahme kann Übelkeit und Erbrechen unter hochgradig emetogenen \\ Chemotherapien wirkungsvoll verhindern.
}

$\mathrm{N}$ eben Haarausfall gehören Übelkeit und Erbrechen zu den Nebenwirkungen von Chemotherapien, welche die Lebensqualität erheblich beeinträchtigen. In einer randomisierten, doppelblind geführten Phase-III-Studie erhielten die Teilnehmer entweder das Neuroleptikum Olanzapin oder Placebo, jeweils in Kombination mit Dexamethason, Aprepitant oder Fosaprepitant und einem 5-HT3Rezeptor-Antagonisten. Von den 380 aufgenommenen Patienten erhielten 192 an den Tagen 1-4 10 mg Olanzapin oral. 188 bekamen Placebo. Der Anteil von Patienten ohne Übelkeit in den ersten 24 Stunden nach Applikation der Chemotherapie war in der Olanzapin-Gruppe mit $74 \%$ signifikant größer als unter Placebo mit 24\%. Dieser Effekt setzte sich in statistisch signifikanter Weise über den Zeitraum von 25-120 Stunden nach der Chemotherapie fort. Auch der sekundäre Endpunkt eines vollständigen Ansprechens - gar keine Übelkeit und keine Anwendung von Akutmedikamenten - fiel mit 86 vs. $65 \%$ bzw. 67 vs. $52 \%$ für die ge- nannten Zeiträume zugunsten von Olanzapin aus. Die wichtigste Nebenwirkung von Olanzapin war ein sedierender Effekt, über den 5\% der Patienten in der Verumgruppe am Tag 2 berichteten.

\section{KOMMENTAR}

Der eindrucksvolle Effekt von Olanzapin am ersten Tag nach der Chemotherapie nutzt sich zwar über die gesamte Beobachtungszeit hinweg etwas $a b$ - doch dürfte jeder einzelne Tag, den sie ohne oder nur mit leichter Übelkeit verbringen, von den betroffenen Patienten als wahrer Segen wahrgenommen werden.

Prof. Dr. med. H. S. FüeßI

Navari RM, Qin R, Ruddy KJ et al. Olanzapine for the prevention of chemotherapy-induced nausea and vomiting. N.Engl J Med. 2016;375:134-42

\title{
Tumorkachexie: Ernährungstherapie senkt Morbidität und Mortalität
}

\begin{abstract}
Eine wichtige supportive Maßnahme bei Tumorpatienten ist die Ernährungstherapie. Sie sollte so früh wie möglich einsetzen; denn dadurch wird nicht zuletzt auch das Ansprechen auf die Therapie und somit die Prognose verbessert.
\end{abstract}

ewichtsabnahme und Mangelernährung sind ein häufiges Problem bei Tumorpatienten. „Dass eine Malnutrition die Prognose verschlechtert, dafür gibt es eindeutige Daten“, betonte Prof. Jann Arends, Freiburg/Br. So sinkt die 5-Jahres-Überlebensrate bei Patienten mit einem Kopf-Hals-Tumor, die eine Radio-/Chemotherapie erhalten, von 71 auf $42 \%$, wenn sie mehr als $10 \%$ des Körpergewichts abnehmen. Entscheidend für die Prognose ist die Gewichtsabnahme vor Einleitung der Therapie, weniger der Gewichtsverlust unter der Therapie.
Der mit der Mangelernährung einhergehende Abbau der Muskelmasse verringert die Kraft und die physische Leistungsfähigkeit. Dazu kommen eine erhöhte Infektanfälligkeit, Störungen der Wundheilung, schlechteres Ansprechen auf die Chemotherapie, eine höhere Rate an Nebenwirkungen und eine Verlängerung des Krankenhausaufenthaltes. Wenn eine Mangelernährung diagnostiziert wird, muss sie behandelt werden, um einen weiteren Gewichtsverlust zu vermeiden. „Eine Ernährungsberatung allein reicht nicht, es muss dann schon eine intensive Ernährungstherapie einsetzen“, so Arends. Grundsätzlich gilt: oral vor Sonde und enteral vor parenteral. Dabei sollte auch auf eine ausreichende Zufuhr von Vitaminen und Spurenelementen geachtet werden. Dagegen werden Antioxidanzien, Glutamin und Probiotika nicht empfohlen, da für diese Substanzen keine ausreichende Evidenz vorliegt. Eine Sondenernährung sollte nur dann eingeleitet werden, wenn z.B. bei einer Obstruktion im oberen Gastrointestinaltrakt eine orale Nahrungsaufnahme nicht möglich ist. „Ist eine Sonde nötig, sollte sie frühzeitig gelegt werden“, so Arends. Ansonsten sei es auch wichtig, den Schluckreflex zu trainieren und zu unterstützen.

Dr. med. Peter Stiefelhagen

Vortrag: The role of nutritional support during radio-/chemotherapy, im Rahmen des ESMO 2016, 7.10.2016 in Kopenhagen 